\title{
Research Progress in Skin Barrier Function
}

\author{
Zinan An \\ University of Delaware, 210 South College Ave. Newark, DE 19716, USA.
}

Keywords: Skin barrier; Barrier structure; Function.

\begin{abstract}
The skin barrier is mainly composed of keratinized envelope and lipid membrane, intermediate filament polymerized protein, keratin, keratin desmosome, lamellar bodies, and stratum corneum keratinocyte. Its function is to prevent the loss of water and prevent external aggression and maintain a steady state in the body. The combination of genetic mutations, allergens, microorganisms, and ultraviolet rays can cause abnormal skin barrier structure, metabolism, and function, causing skin diseases such as ichthyosis and atopic dermatitis. Clarify the link between skin diseases and barrier structure and functional abnormalities and guide the development of effective diagnostic and preventive measures.
\end{abstract}

\section{Introduction}

The skin is an organ in direct contact with the outside world and the first barrier to the immune system. On the one hand, it can organize external adverse factors to enter and harm the human body to a certain extent; On the other hand, it can prevent the loss of moisture and lipids in the human epidermis and dermis, thereby maintaining the stability of the skin barrier function. The barrier function of the stratum corneum plays a vital role. Atopic dermatitis, psoriasis, and various skin diseases such as contact dermatitis are related to the stabilization of skin barrier function. Therefore, skin barrier function has always been a hot topic in dermatology clinical work. We will do an overview of skin structure, skin barrier and skin disease.

\section{Stratum Corneum Structure}

The skin is the largest organ in the human body. The epidermis is the outermost layer of the skin, which is mainly composed of Keratinocyte, melanocytes, Langerhans cells, and Merkel cells. Keratinocyte is the main constituent cells of epidermis, and the number of cells accounts for more than $80 \%$ of the epidermal cells. The foremost structural basis of skin barrier function is the stratum corneum formed by the most surface Keratinocyte, the lipids between the keratinocytes, the natural protective factors (NMF), and so on.

\subsection{Keratinocyte}

Keratinocytes undergo cell mitosis, differentiation, degradation until they disappear. They go through the basal layer, spinous cell layer, and granular cell layer, and eventually become the outermost cuticle of the epidermis, forming its unique multilayer structure. Keratinocytes are "stacked" in the continuous matrix of intercellular lipids, forming the classic "brick-wall" organizational system [1].

\subsubsection{Keratin}

Keratinocytes are the main cells that make up the epidermis. Keratin is the main gene product of keratinocytes. This type of protein forms the intermediate intercellular filaments that make cells elastic. Normal epidermal basal layer cells are undifferentiated state and have the ability to divide and proliferate. Normal epidermal basal layer cells are undifferentiated, have the ability to divide and proliferate, and specifically express keratin 5(K5) / keratin 14(K14). Once cells enter the spiny cell 
layer, the expression of the specific differentiated protein keratin 1 (K1) / keratin 10 (K10) can be detected [2, 3].

\subsubsection{Intermediate Filament Protein}

Intermediate filament protein is made up of components that are structured like cytoskeletal that exist in the cells of vertebrates and an uncountable number of invertebrates. Intermediate filaments are self-possessed of a family of related proteins sharing mutual structural and arrangement features. They acquired prefix intermediate since their average diameter, which is $10 \mathrm{~nm}$, exists between those of narrower microfilaments called actin and wider myosin filaments that are found in muscle cells. Currently, the width of intermediate filament is associated with the span of actin microfilaments, which is $7 \mathrm{~nm}$ or the diameter of microtubules that is $25 \mathrm{~nm}$. Intermediate filament protein is categorized into six subgroups basing on two factors, one the similarity that exists in the sequence amino acid and the structure of the protein. Contrary to microtubules, the distribution cell of Intermediate filament indicates no good correlation with the distribution of either endoplasmic reticulum or mitochondria [4].

Intermediate filament protein has biomechanical properties that enable it to play major role skin barrier function. Intermediate filaments are relatively deformable protein that has the capabilities of stretching their initial length numerous times [5]. The adaptational feature behind this ability is from their large deformation resulting from their hierarchical structure. The hierarchical erection facilitates a fallen activation of the distortion mechanism at a wide range degree of strain. At first, the coupled alpha-helices of piece-length filaments untwist when they are strained, then when the straining force increases, they convert into beta-sheets. Finely, when there is increased strain, the hydrogen bonds that exist between beta-sheets slips and the monomers of UFL slide along with one another.

The combination of intermediate filaments, microtubules, and actin filament built up vital component of the cytoskeleton. In that amalgamation, they can provide mechanical stability of tissues and donate to countless cellular processes like the positioning of the organelle, trafficking of vesicle, regulation, cycle regulation of cell, and ensures motility of cells. Besides, intermediate filaments provide a significant cushioning system of protection against several kinds of stressors ranging from physical, microbial, or chemical ones. Intermediate filaments create a sophisticated three-dimension network with subcellular planning that delivers a barrier function in a simple and stratified epithelium. The Keratin intermediate filament is subapically augmented in a dense network of filament within the simple epithelium of the intestine. The keratin intermediate filament of stratified cornea epithelium develops a dense three-dimension network that crisscrosses the entire cytoplasm and is involved in desmosome at cell-cell contact sites. Thus, releasing fragments of Keratin that have antibacterial properties into the tear fluid.

\subsection{Intercellular Lipid}

Intercellular lipids exist in human stratum in corneum fundamentally consisting of fatty acid, cholesterol, sphingolipids like ceramides and cholesteryl sulfate. Intercellular lipids form wide and multicellular spaces, which are vital in fulfilling the regulatory duty. Intercellular lipids have both physical and chemical attributes that leads to the construction and functioning that facilitate the skin's absorbency barricade. Lipids accrue in micro-organelles referred to as lamellar granules which is epidermal keratinocytes discriminate. This kind of lipid is extruded hooked on the intercellular gaps where it experiences enzymatic dispensation that will lead to the production of lipid combination containing of cholesterol, ceramides, and fatty acids. This kind of intercellular lipids is exceptionally prearranged into multilamellar multifaceted that seals utmost of the intercellular interstellar of the stratum corneum. The blockade chattels that stratum corneum poses are driven by the phase attributes of intercellular lipids [6]. The intercellular lipids that have an unusual structure called acyl glucosyl ceramide play a significant duty in assembling of the lamellar granules. In addition, acyl-ceramides do a substantial stimulus on the alignment of the lamellae within stratum corneum. 
Thus, intercellular lipids provide the skin with the ability to act as a barrier between the body and the surroundings. The protective nature of the skin is attributed to the lipid composition that is found in the external skin layer that builds up the stratum cornea. The intercellular lipid composition of the stratum cornea has adopted a complexly ordered three-dimension structure fixed densely into lipid layers called lamellae. The structural alignment of intercellular in stratum cornea ensures that the skin doesn't lose water excessively via the epidermis, and to ensure that no environmental elements that can penetrate the worthwhile epidermal and dermal layers that will lead to provocation of immune reaction.

\subsection{Cornified Envelope (CE)}

The cornified envelope is an edifice that is formed underneath the plasma sheath in lethally differentiating stratified squamous epithelia. As keratinocytes advances towards the upper epidermis, they go through an exclusive process of cell death called cornification. The Cornification process involves the cross-connecting of keratinocyte protein like involucrin and loricrin through transglutaminases and the cessation of the nucleus and additional organelles via intracellular and veiled proteases. The process of breaking down of the nucleus and more organelle is controlled by the $\mathrm{Ca} 2+$ concentration rise that is found in the epidermis. The keratinocyte proteins, Involucrin and Loricrin, are prearranged in the epidermal variation composite that is linked to a massive number of genes indoctrination nonterminated components of Cornified Envelope. The keratinocytes release specific proteins and lipids that are significant in the development of the cornified envelope. The facilitated dense cross-joining layers of submembrane that deliberate rigidity to the upper epidermis. This creates a room for keratin filament to attach to any position in the cell membrane and thus to act as a water-impervious barrier.

The cornified Envelope provides a crucial physical barrier squamous epithelium in mammals as it has a $10 \mathrm{~nm}$ dense layer that is greatly crosslinked by insoluble proteins. When it comes to the specific situation of the epidermis, it is coated by a $5 \mathrm{~nm}$ thick layer of ceramide lipid that is covalently bonded to the protein. The combination of extracellular lipids and ordered lamellae, together with the cornified envelop that is built around extracellular lipids, are of relevance in ensuring effective physical and aquatic barrier purposes of the skin. Besides, when the desmosomes get crosslinked to the cornified envelope, they form corneodesmosomes, which are essential in binding cells that are cornified together [7]. A mature, incurably cornified cell that is differentiated consists majorly of keratin filaments that are covalently bonded to the cornified envelope in lamellae lipid. The precise configuration of the cornified envelop tends to shift depending on the epithelia, which is the comparative amino-acid conformation of the proteins used, which controls different mechanical properties. Therefore, the epidermis which plays the role of acting as a barrier against the elements of the environment, it does so by methods of numerous layers of critically discerned dead keratinocytes, which are cornified layers. Thus, the cornified envelope takes place for the plasma membrane that was initially made up of keratins that are programmed within an unfathomable amalgam of protein. Just like any other skin segment that is dedicated to protecting the inner internal parts, the cornified envelope has developed features that make it effectively act as a barrier for many environmental elements.

\section{The Regulating Factors of Epidermal Barrier}

The function of epidermal barrier mainly includes physical, chemical, biological and water permeability. The level of epidermal barrier function can reflect the severity of some skin diseases and regulate the biological function of skin. Epidermal barrier function is regulated by several factors, such as ions, enzymes, and lipids.

\subsection{Calcium}

The relative concentration of ions inside and outside in keratinocyte envelope (i.e. envelope polarity) plays an major role in the of skin barrier repair process. The concentration of intracellular 
free calcium is about $100 \mathrm{nmol} / \mathrm{L}$, and that of extracellular calcium is about $1-3 \mathrm{nmol} / \mathrm{L}$. However, maintaining the difference between the concentration of intracellular and external ions is mainly maintained by ion-related channels. Calcium ions induce keratinocytes to differentiate into the stratum corneum, proliferate, form the keratinocyte sheath, and synthesize the epidermal lipid [8]. The artificially cultured Keratinocytes have a rapid proliferation state with extracellular calcium ions concentration of 0.02-0.1 mmol/L. Some studies have found that fluctuations in Ca2+ concentration can also affect the expression of LOR, IVL, and sapogenin (precursors of FLG), so extracellular $\mathrm{Ca} 2+$ concentration is crucial for the formation of skin barrier function [9, 10]. Increasing intracellular and extracellular calcium ion concentrations can cause depolarization of the envelope, disturbing the inward movement of the envelope and exocytosis of the laminar body, thereby affecting the formation of the stratified lamellar membrane between the cells and delaying the repair of the skin barrier. A concentration of 0.01 to $1.80 \mathrm{mmol} / \mathrm{L}$ can significantly inhibit the restoration of skin barrier function. Within this concentration, the degree of inhibition is directly proportional to the concentration of calcium ions [11].

\subsection{Enzymes}

The animals, plants, and microorganisms we come into contact with in the outward environment produce or contain proteases. From genetic point perspective, environmental protease and endogenous protease lead to the lack or damage of skin barrier function and enhance the sensitivity of the body to allergens, which further aggravate allergic diseases [12]. Human tissue kallikreins (KLKs) regulate the homeostasis of the skin barrier function. It can hydrolyze proteins to avoid peeling, bacterial invasion and lipid permeation. There is increasing evidence that KLKs can be involved by activating protease receptor signaling Inflammation of the skin, pigmentation, and inhibition of skin tumors [13].

\section{Cuticle-related Barrier Function}

Cuticle-related barrier function means the water permeability barrier function, which mainly process in the cuticle, thus, it is also called the cuticle barrier function. This important function is produced by keratinocyte and the lipid mixture between them.

\subsection{Tape Stripping on Trans epidermal Water Loss (TEWL)}

Various internal and external factors can affect the skin's permeability barrier, such as the climate, physical stress, and many skin systemic diseases. The stratum corneum is the result of terminal differentiation of the epidermis and protects the body. TEWL reflects the amount of water evaporated from the skin surface, so it is an essential indicator for evaluating skin function. Some scholars have studied the isolated skin model under the conditions of standard laboratory temperature $25^{\circ} \mathrm{C}$ and relative humidity of $40 \%$ to $50 \%$, and evaluated the relationship between TEWL size of the skin and the skin integrity. The thickness has a negative correlation with the measured TEWL size (correlation coefficient $=0.45$ ), and the difference is statistically significant $(P=0.007)$ [14], which is consistent with the conclusion previously reported by Sekkat. Therefore, it can be considered that the growth thickness of the skin stratum corneum is inversely related to the skin TEWL, that is, the thicker the skin stratum corneum grows, the smaller the TEWL value of the skin, the more perfect the skin barrier function, and vice versa. Repeated tape stripping of the dog's skin caused damage to the stratum corneum, and an increase in TEWL was detected, suggesting impaired skin barrier function [15].

\subsection{Antimicrobial and Immune Barrier}

The skin has an outer layer known as the epidermis functioning as a physical protective layer against pathogens. Consequently, a rupture in the skin caused by an injury poses a threat because of the introduction of numerous microbes to the injured skin [16]. Significantly, once there is an injury on the epidermis, the innate immunes system and associated effectors will play take part in the 
protection of an individual against cutaneous and systemic infections. Notably, the major elements of innate immune system are phagocytic cells including natural killer (NK) cells, mast cells, basophils, and eosinophil [17]. The skin has epidermal keratinocytes which play a key role in the healing of a wound. Significantly, epidermal keratinocytes make up $90 \%$ of all cells found in this layer of the skin. Additionally, these keratinocytes have a sensing mechanism in which it makes the skin be in a position to detect pathogenic patterns being expressed by both hosts and associated host danger molecules. When there are microbes in the skin, there will be activation of keratinocyte hence leading to release of inflammatory cytokines together with antimicrobial molecules.

Significantly, when it comes to antimicrobial and immune barrier, the skin has an immune response helping in recognizing any possible pathogens. In the skin, the immune system is able to recognize PAMPs present on microbes or pathogens. The recognition of microbes on the skin is usually done by germline-encoded pattern recognition receptors commonly referred to as PRR. Notably, there are four fundamental groups of germline recognition receptors. They include: toll-like receptors, nucleotide-binding oligomerization domain-like receptors (NLRs), retinoic acid-inducible gene receptors and c-type lectin receptors commonly abbreviated as CLR. An example of how recognition of pathogens takes place can be given by: where there is an injury on the skin, there will be easier penetration of viruses and pathogenic microbes into the epidermis. PAMPs and DAMPs facilitate the release of pro-inflammatory cytokines. In the skin, pro-inflammatory cytokines are IL-1 $\beta$, TNF $\alpha$, and IFN $\gamma$ [16]. The next step will involve recruitment of neutrophils and macrophages by the inflammatory cytokines to the place where the injury occurred on the skin.

There are antibacterial and antiviral proteins being produced inside the body that assist the inborn immune system to annihilate microbes. Illustratively, keratinocytes produce antimicrobial proteins that infiltrate immune cells hence preventing further infection. The antimicrobial mechanisms enhancing skin protection include inhibition of bacterial protein and DNA systems. Additionally, when it comes to antifungal mechanisms of AMPs, there is disruption of the fungal mitochondrion membrane. It is significant to note that, there are two classes of AMPs. They include: cathelicidins and defensins. These two classes of AMPs help in the provision of a wider spectrum protection against grams bacteria. As an illustration, the cathelicidins LL-37 has direct potency of antibacterial activity against Group A bacteria. When it comes to human defensins, there is HbD1 which are expressed in the epithelial cells. Additionally, hBD2 are usually expressed in low levels with a rapid proliferation in in their production where there is an injury or irritation on the skin [18].

In the skin, there are commensal organisms which promote annihilation of pathogenic bacteria and virus. Significantly, these commensal organisms improve the healing process of the skin where the injury occurred [16]. An example of these organisms includes S. epidermidis which helps in the production of AMPs that acts together with endogenous AMPs producing keratinocytes. There are also peptides being produced by S. epidermidis. An example includes the phenol-soluble modulins.

\section{AD and Skin Barrier Function}

Atopic dermatitis (AD) is a chronic pruritic inflammatory skin disease, characterized by abnormal regulation of Th1 and Th2 cells, extremely active IgE and mast cells, and signal transduction by dendritic cells. As well as the permeability barrier, the antimicrobial barrier was impaired in AD patients. Colonization of Staphylococcus aureus is a shared feature of $\mathrm{AD}$, and the colonization of its superantigen product makes AD further worsened by producing a large amount of IgE and exotoxin. In addition, AD patients are susceptible to a wide range of skin viral infections, including contact with molluscum contagiosum, herpes simplex (Kaposi), and life-threatening cowpox [19-21]. Extensive skin fungal and Malassezia infections can also occur in AD patients, who also stimulate specific IgE production like $\mathrm{S}$. aureus. These findings indicate that $\mathrm{AD}$ patients lack an intact antimicrobial barrier. Moreover, AD not only has permeability and antibacterial dysfunction, but also these two functions are mutually restricted and interdependent [20]. Barriers to osmotic barriers are vulnerable to secondary infections. Conversely, colonization and infection of pathogens further worsen barrier function. In patients with $\mathrm{AD}$, the susceptibility to pathogens after the osmotic barrier 
is impaired is caused not only by an increase in the $\mathrm{pH}$ of the skin surface, but also by a decrease in antibacterial active substances such as FFA, ceramide metabolites, and sphingosine [22].

\section{Conclusion}

The skin is the most vital organ that protects the human body system. Being the largest part of the body system and the outermost layer of the body, it plays a protective role for all the body parts. In its proactive role, the skin is helped by the epidermal keratinocytes, which form $90 \%$ of the skin cells. Through the use of epidermal keratinocytes, the skin can heal wounds after an injury, which acts as entry points for microbes that lead to diseases such as Atopic dermatitis. Atopic dermatitis is among the skin diseases that predispose patients to with molluscum contagiosum, herpes simplex (Kaposi), and life-threatening cowpox.

Despite the skin's protective role, it also has environmental proteases, enzymes that warns the body against allergies that cause allergic diseases. The skin also acts in a cuticle-related barrier function that prevents the body from excessive water loss. Keratin intermediate filament has antibacterial properties in the tear fluid. Therefore, it is evident makes sure that all the body parts are protected through ensuring sufficient water in the body and prevention of injuries that act as windows for entry of microbes and other harmful molecules in the body system. Hence, all these protection roles of the skin help in immune system boosting, thus preventing further multiplication of infections in the human body.

\section{References}

[1] Harding CR. The stratum corneum: structure and function in health and disease. Dermatol Ther. 2004; 17 Suppl 1: 6-15.

[2] Bloor BK, Tidman N, Leigh IM, Odell E, Dogan B, Wollina U, et al. Expression of keratin K2e in cutaneous and oral lesions: association with keratinocyte activation, proliferation, and keratinization. Am J Pathol. 2003; 162: 963-75.

[3] Leigh IM, Purkis PE, Whitehead P, Lane EB. Monospecific monoclonal antibodies to keratin 1 carboxy terminal (synthetic peptide) and to keratin 10 as markers of epidermal differentiation. $\mathrm{Br} \mathrm{J}$ Dermatol. 1993; 129: 110-9.

[4] San José GPG, M.T. Vázquez, F.J. Naves, I. Esteban and J.A. Vega. Intermediate filament proteins in developing and adult human dorsal root and sympathetic ganglia. Eur J Anat. 2001; 5: 9.

[5] Preisner H, Habicht J, Garg SG, Gould SB. Intermediate filament protein evolution and protists. Cytoskeleton (Hoboken). 2018; 75: 231-43.

[6] Candi E, Tarcsa E, Digiovanna JJ, Compton JG, Elias PM, Marekov LN, et al. A highly conserved lysine residue on the head domain of type II keratins is essential for the attachment of keratin intermediate filaments to the cornified cell envelope through isopeptide crosslinking by transglutaminases. Proc Natl Acad Sci U S A. 1998; 95: 2067-72.

[7] Swartzendruber DC, Wertz PW, Kitko DJ, Madison KC, Downing DT. Molecular models of the intercellular lipid lamellae in mammalian stratum corneum. J Invest Dermatol. 1989; 92: 251-7.

[8] Denda M, Inoue K, Fuziwara S, Denda S. P2X purinergic receptor antagonist accelerates skin barrier repair and prevents epidermal hyperplasia induced by skin barrier disruption. J Invest Dermatol. 2002; 119: 1034-40.

[9] Elias PM, Menon GK. Structural and lipid biochemical correlates of the epidermal permeability barrier. Adv Lipid Res. 1991; 24: 1-26. 
[10] Sun XD, You Y, Zhang L, Zheng S, Hong Y, Li J, et al. The possible role of TRPC6 in atopic dermatitis. Med Hypotheses. 2012; 78: 42-4.

[11] Denda M, Fuziwara S, Inoue K. Influx of calcium and chloride ions into epidermal keratinocytes regulates exocytosis of epidermal lamellar bodies and skin permeability barrier homeostasis. J Invest Dermatol. 2003; 121: 362-7.

[12] Takai T, Ikeda S. Barrier dysfunction caused by environmental proteases in the pathogenesis of allergic diseases. Allergol Int. 2011; 60: 25-35.

[13] Eissa A, Diamandis EP. Human tissue kallikreins as promiscuous modulators of homeostatic skin barrier functions. Biol Chem. 2008; 389: 669-80.

[14] Hui X, Elkeeb R, Chan H, Maibach HI. Ability to estimate relative percutaneous penetration via a surrogate maker - trans epidermal water loss? Skin Res Technol. 2012; 18: 108-13.

[15] Shimada K, Yoshihara T, Yamamoto M, Konno K, Momoi Y, Nishifuji K, et al. Transepidermal water loss (TEWL) reflects skin barrier function of dog. J Vet Med Sci. 2008; 70: 841-3.

[16] Coates M, Blanchard S, MacLeod AS. Innate antimicrobial immunity in the skin: A protective barrier against bacteria, viruses, and fungi. PLoS Pathog. 2018; 14: e1007353.

[17] MacLeod AS, Mansbridge JN. The Innate Immune System in Acute and Chronic Wounds. Adv Wound Care (New Rochelle). 2016; 5: 65-78.

[18] Plato A, Hardison SE, Brown GD. Pattern recognition receptors in antifungal immunity. Semin Immunopathol. 2015; 37: 97-106.

[19] Kang KF, Tian RM. Atopic dermatitis. An evaluation of clinical and laboratory findings. Int J Dermatol. 1987; 26: 27-32.

[20] Leung DY, Boguniewicz M, Howell MD, Nomura I, Hamid QA. New insights into atopic dermatitis. J Clin Invest. 2004; 113: 651-7.

[21] Novak N, Bieber T, Leung DY. Immune mechanisms leading to atopic dermatitis. J Allergy Clin Immunol. 2003; 112: S128-39.

[22] Sator PG, Schmidt JB, Honigsmann H. Comparison of epidermal hydration and skin surface lipids in healthy individuals and in patients with atopic dermatitis. J Am Acad Dermatol. 2003; 48: 352-8. 